\title{
Potential sensitivity of photosynthesis and isoprene emission to direct radiative effects of atmospheric aerosol pollution
}

\section{Susanna Strada and Nadine Unger}

Correspondence to: Susanna Strada (susanna.strada@1sce.ipsl.fr)

The copyright of individual parts of the supplement might differ from the CC-BY 3.0 licence. 
Table S1. Absolute and percent changes in regional annual average of aerosol column burden (ACB) as simulated by NASA ModelE2-YIBs for sulfate $\left(\mathrm{SO}_{4}\right)$, nitrate and secondary organic aerosol (SOA) in: eastern North America, Eurasia, north-eastern China, north-western Amazon Basin and central Africa (see green boxes on Fig. 1a in the manuscript). Changes are computed between the control experiment (SimCTRL) and sensitivity experiments: SimNOant, without all anthropogenic emissions; SimNObb, without biomass burning emissions; and SimNOind, without anthropogenic emissions except biomass burning. The acronym "ns" indicates differences that are not statistically significant at the $95 \%$ confidence level (based on a Student's t-test).

\begin{tabular}{|c|c|c|c|c|}
\hline Region & Species & SimCTRL - SimNOant & SimCTRL - SimNObb & SimCTRL - SimNOind \\
\hline Eastern & $\mathrm{SO}_{4}$ & $3.24 \mathrm{mg} \mathrm{m}^{-2}$ & $-0.14 \mathrm{mg} \mathrm{m}^{-2}$ & $3.25 \mathrm{mg} \mathrm{m}^{-2}$ \\
\hline \multirow{5}{*}{$\begin{array}{l}\text { North America } \\
\left(70^{\circ}-100^{\circ} \mathrm{W} ; 36^{\circ}-52^{\circ} \mathrm{N}\right)\end{array}$} & & $73.26 \%$ & $3.26 \%$ & $73.54 \%$ \\
\hline & $\mathrm{NO}_{3}$ & $15.02 \mathrm{mg} \mathrm{m}^{-2}$ & $3.44 \mathrm{mg} \mathrm{m}^{-2}$ & $12.72 \mathrm{mg} \mathrm{m}^{-2}$ \\
\hline & & $93.19 \%$ & $21.36 \%$ & $78.88 \%$ \\
\hline & SOA & $2.14 \mathrm{mg} \mathrm{m}^{-2}$ & $0.39 \mathrm{mg} \mathrm{m}^{-2}$ & $0.87 \mathrm{mg} \mathrm{m}^{-2}$ \\
\hline & & $78.87 \%$ & $14.34, \%$ & $31.90 \%$ \\
\hline \multirow{6}{*}{$\begin{array}{l}\text { Eurasia } \\
\left(-10^{\circ} \mathrm{W}-80^{\circ} \mathrm{E} ; 40^{\circ}-65^{\circ} \mathrm{N}\right)\end{array}$} & $\mathrm{SO}_{4}$ & $2.60 \mathrm{mg} \mathrm{m}^{-2}$ & $-0.08 \mathrm{mg} \mathrm{m}^{-2}$ & $2.69 \mathrm{mg} \mathrm{m}^{-2}$ \\
\hline & & $64.70 \%$ & $-2.11 \%$ & $67.05 \%$ \\
\hline & $\mathrm{NO}_{3}$ & $14.28 \mathrm{mg} \mathrm{m}^{-2}$ & $3.35 \mathrm{mg} \mathrm{m}^{-2}$ & $11.95 \mathrm{mg} \mathrm{m}^{-2}$ \\
\hline & & $93.65 \%$ & $21.97 \%$ & $78.36 \%$ \\
\hline & SOA & $2.05 \mathrm{mg} \mathrm{m}^{-2}$ & $0.30 \mathrm{mg} \mathrm{m}^{-2}$ & $1.39 \mathrm{mg} \mathrm{m}^{-2}$ \\
\hline & & $77.06 \%$ & $11.16 \%$ & $52.18 \%$ \\
\hline North-eastern & $\mathrm{SO}_{4}$ & $3.77 \mathrm{mg} \mathrm{m}^{-2}$ & ns & $3.81 \mathrm{mg} \mathrm{m}^{-2}$ \\
\hline \multirow{5}{*}{$\begin{array}{l}\text { China } \\
\left(100^{\circ}-120^{\circ} \mathrm{E} ; 10^{\circ}-35^{\circ} \mathrm{N}\right)\end{array}$} & & $77.29 \%$ & ns & $78.22 \%$ \\
\hline & $\mathrm{NO}_{3}$ & $8.42 \mathrm{mg} \mathrm{m}^{-2}$ & $1.97 \mathrm{mg} \mathrm{m}^{-2}$ & $7.06 \mathrm{mg} \mathrm{m}^{-2}$ \\
\hline & & $85.56 \%$ & $20.03 \%$ & $71.72 \%$ \\
\hline & SOA & $2.01 \mathrm{mg} \mathrm{m}^{-2}$ & $0.32 \mathrm{mg} \mathrm{m}^{-2}$ & $1.38 \mathrm{mg} \mathrm{m}^{-2}$ \\
\hline & & $86.31 \%$ & $13.77, \%$ & $59.13 \%$ \\
\hline North-western & $\mathrm{SO}_{4}$ & $0.19 \mathrm{mg} \mathrm{m}^{-2}$ & $0.04 \mathrm{mg} \mathrm{m}^{-2}$ & $0.21 \mathrm{mg} \mathrm{m}^{-2}$ \\
\hline Amazon Basin & & $16.88 \%$ & $3.83 \%$ & $18.46 \%$ \\
\hline \multirow[t]{4}{*}{$\left(73^{\circ}-65^{\circ} \mathrm{W} ; 5^{\circ} \mathrm{S}-5^{\circ} \mathrm{N}\right)$} & $\mathrm{NO}_{3}$ & $3.91 \mathrm{mg} \mathrm{m}^{-2}$ & $1.48 \mathrm{mg} \mathrm{m}^{-2}$ & $3.14 \mathrm{mg} \mathrm{m}^{-2}$ \\
\hline & & $78.76 \%$ & $29.87 \%$ & $63.22 \%$ \\
\hline & SOA & $1.81 \mathrm{mg} \mathrm{m}^{-2}$ & $0.54 \mathrm{mg} \mathrm{m}^{-2}$ & $0.55 \mathrm{mg} \mathrm{m}^{-2}$ \\
\hline & & $79.94 \%$ & $23.70 \%$ & $29.19 \%$ \\
\hline \multirow{6}{*}{$\begin{array}{l}\text { Central Africa } \\
\left(10^{\circ}-25^{\circ} \mathrm{E} ; 10^{\circ} \mathrm{S}-5^{\circ} \mathrm{N}\right)\end{array}$} & $\mathrm{SO}_{4}$ & $1.05 \mathrm{mg} \mathrm{m}^{-2}$ & $0.58 \mathrm{mg} \mathrm{m}^{-2}$ & $0.72 \mathrm{mg} \mathrm{m}^{-2}$ \\
\hline & & $56.48 \%$ & $29.97 \%$ & $39.91 \%$ \\
\hline & $\mathrm{NO}_{3}$ & $6.53 \mathrm{mg} \mathrm{m}^{-2}$ & $2.98 \mathrm{mg} \mathrm{m}^{-2}$ & $4.75 \mathrm{mg} \mathrm{m}^{-2}$ \\
\hline & & $82.12 \%$ & $37.50 \%$ & $59.63 \%$ \\
\hline & SOA & $4.42 \mathrm{mg} \mathrm{m}^{-2}$ & $2.14 \mathrm{mg} \mathrm{m}^{-2}$ & $1.10 \mathrm{mg} \mathrm{m}^{-2}$ \\
\hline & & $89.97 \%$ & $43.62, \%$ & $22.30 \%$ \\
\hline
\end{tabular}


Table S2. As Table S1 for absolute and percent changes in regional annual effective radiative forcing (ERF).

\begin{tabular}{|c|c|c|c|c|}
\hline Region & Species & SimCTRL - SimNOant & SimCTRL - SimNObb & SimCTRL - SimNOind \\
\hline Eastern & $\mathrm{SO}_{4}$ & $-1.06 \mathrm{~W} \mathrm{~m}^{-2}$ & $0.04 \mathrm{~W} \mathrm{~m}^{-2}$ & $-1.05 \mathrm{~W} \mathrm{~m}^{-2}$ \\
\hline \multirow{5}{*}{$\begin{array}{l}\text { North America } \\
\left(70^{\circ}-100^{\circ} \mathrm{W} ; 36^{\circ}-52^{\circ} \mathrm{N}\right)\end{array}$} & & $78.97 \%$ & $2.72 \%$ & $78.37 \%$ \\
\hline & $\mathrm{NO}_{3}$ & $-1.29 \mathrm{~W} \mathrm{~m}^{-2}$ & $-0.27 \mathrm{~W} \mathrm{~m}^{-2}$ & $-1.10 \mathrm{mg} \mathrm{m}^{-2}$ \\
\hline & & $93.90 \%$ & $19.76 \%$ & $79.90 \%$ \\
\hline & SOA & $-0.23, \mathrm{~W} \mathrm{~m}^{-2}$ & $0.05 \mathrm{~W} \mathrm{~m}^{-2}$ & $0.08 \mathrm{~W} \mathrm{~m}^{-2}$ \\
\hline & & $72.55 \%$ & $15.94, \%$ & $25.34 \%$ \\
\hline \multirow{6}{*}{$\begin{array}{l}\text { Eurasia } \\
\left(-10^{\circ} \mathrm{W}-80^{\circ} \mathrm{E} ; 40^{\circ}-65^{\circ} \mathrm{N}\right)\end{array}$} & $\mathrm{SO}_{4}$ & $-0.86 \mathrm{~W} \mathrm{~m}^{-2}$ & $0.02 \mathrm{~W} \mathrm{~m}^{-2}$ & $-0.86 \mathrm{~W} \mathrm{~m}^{-2}$ \\
\hline & & $70.79 \%$ & $-1.70 \%$ & $71.02 \%$ \\
\hline & $\mathrm{NO}_{3}$ & $-1.25 \mathrm{~W} \mathrm{~m}^{-2}$ & $-0.26 \mathrm{~W} \mathrm{~m}^{-2}$ & $-1.05 \mathrm{~W} \mathrm{~m}^{-2}$ \\
\hline & & $94.79 \%$ & $19.79 \%$ & $79.69 \%$ \\
\hline & SOA & $-0.23 \mathrm{~W} \mathrm{~m}^{-2}$ & $-0.04 \mathrm{~W} \mathrm{~m}^{-2}$ & $-0.15 \mathrm{~W} \mathrm{~m}^{-2}$ \\
\hline & & $71.31 \%$ & $11.73 \%$ & $45.34 \%$ \\
\hline North-eastern & $\mathrm{SO}_{4}$ & $-1.43 \mathrm{~W} \mathrm{~m}^{-2}$ & ns & $-1.43 \mathrm{~W} \mathrm{~m}^{-2}$ \\
\hline \multirow{5}{*}{$\begin{array}{l}\text { China } \\
\left(100^{\circ}-120^{\circ} \mathrm{E} ; 10^{\circ}-35^{\circ} \mathrm{N}\right)\end{array}$} & & $81.71 \%$ & ns & $82.09 \%$ \\
\hline & $\mathrm{NO}_{3}$ & $-0.74 \mathrm{~W} \mathrm{~m}^{-2}$ & $-0.16 \mathrm{~W} \mathrm{~m}^{-2}$ & $-0.63 \mathrm{~W} \mathrm{~m}^{-2}$ \\
\hline & & $85.76 \%$ & $18.41 \%$ & $73.01 \%$ \\
\hline & SOA & $-0.14 \mathrm{~W} \mathrm{~m}^{-2}$ & $-0.03 \mathrm{~W} \mathrm{~m}^{-2}$ & $-0.10 \mathrm{~W} \mathrm{~m}^{-2}$ \\
\hline & & $77.69 \%$ & $14.35, \%$ & $56.20 \%$ \\
\hline North-western & $\mathrm{SO}_{4}$ & $-0.09 \mathrm{~W} \mathrm{~m}^{-2}$ & $-0.02 \mathrm{~W} \mathrm{~m}^{-2}$ & $-0.09 \mathrm{~W} \mathrm{~m}^{-2}$ \\
\hline Amazon Basin & & $25.63 \%$ & $6.35 \%$ & $25.83 \%$ \\
\hline \multirow[t]{4}{*}{$\left(73^{\circ}-65^{\circ} \mathrm{W} ; 5^{\circ} \mathrm{S}-5^{\circ} \mathrm{N}\right)$} & $\mathrm{NO}_{3}$ & $-0.31 \mathrm{~W} \mathrm{~m}^{-2}$ & $-0.12 \mathrm{~W} \mathrm{~m}^{-2}$ & $-0.25 \mathrm{~W} \mathrm{~m}^{-2}$ \\
\hline & & $78.52 \%$ & $29.69 \%$ & $62.87 \%$ \\
\hline & SOA & $-0.15 \mathrm{~W} \mathrm{~m}^{-2}$ & $-0.06 \mathrm{~W} \mathrm{~m}^{-2}$ & $-0.06 \mathrm{~W} \mathrm{~m}^{-2}$ \\
\hline & & $75.32 \%$ & $28.66 \%$ & $29.53 \%$ \\
\hline \multirow{6}{*}{$\begin{array}{l}\text { Central Africa } \\
\left(10^{\circ}-25^{\circ} \mathrm{E} ; 10^{\circ} \mathrm{S}-5^{\circ} \mathrm{N}\right)\end{array}$} & $\mathrm{SO}_{4}$ & $-0.42 \mathrm{~W} \mathrm{~m}^{-2}$ & $-0.19 \mathrm{~W} \mathrm{~m}^{-2}$ & $-0.28 \mathrm{~W} \mathrm{~m}^{-2}$ \\
\hline & & $65.80 \%$ & $30.33 \%$ & $44.78 \%$ \\
\hline & $\mathrm{NO}_{3}$ & $-0.55 \mathrm{~W} \mathrm{~m}^{-2}$ & $-0.29 \mathrm{~W} \mathrm{~m}^{-2}$ & $-0.38 \mathrm{~W} \mathrm{~m}^{-2}$ \\
\hline & & $83.39 \%$ & $43.45 \%$ & $58.13 \%$ \\
\hline & SOA & $-0.30 \mathrm{~W} \mathrm{~m}^{-2}$ & $-0.16 \mathrm{~W} \mathrm{~m}^{-2}$ & $-0.08 \mathrm{~W} \mathrm{~m}^{-2}$ \\
\hline & & $88.09 \%$ & $47.98, \%$ & $22.91 \%$ \\
\hline
\end{tabular}


Table S3. Absolute and percent changes in seasonal average shortwave visible (SW VIS) solar radiation, canopy temperature, gross primary productivity (GPP), and isoprene emission in: eastern North America, Eurasia and north-eastern China (green boxes on Fig. 1a in the manuscript). Changes are computed between the control experiment (SimCTRL) and sensitivity experiments: SimNOant, without all anthropogenic emissions; SimNObb, without biomass burning emissions; and SimNOind, without anthropogenic emissions except biomass burning. Only boreal summer (JJA) and winter (DJF) seasonal averages are presented. The acronym "ns" indicates differences that are not statistically significant at the $95 \%$ confidence level (based on a Student's t-test).

\begin{tabular}{|c|c|c|c|c|c|c|}
\hline \multirow{2}{*}{$\begin{array}{l}\text { Region } \\
\text { Eastern }\end{array}$} & \multicolumn{2}{|l|}{ Variable } & \multirow{2}{*}{$\begin{array}{l}\text { Season } \\
\text { JJA }\end{array}$} & \multirow{2}{*}{$\begin{array}{c}\text { SimCTRL }- \text { SimNOant } \\
-14.63 \mathrm{~W} \mathrm{~m}^{-2}\end{array}$} & \multirow{2}{*}{$\begin{array}{c}\text { SimCTRL }- \text { SimNObb } \\
-3.15 \mathrm{~W} \mathrm{~m}^{-2}\end{array}$} & \multirow{2}{*}{$\begin{array}{c}\text { SimCTRL }- \text { SimNOind } \\
-4.76 \mathrm{~W} \mathrm{~m}^{-2}\end{array}$} \\
\hline & SW VIS Solar Radiation & Total & & & & \\
\hline North America & & & & $-5.62 \%$ & $-1.21 \%$ & $-1.83 \%$ \\
\hline \multirow[t]{22}{*}{$\left(70^{\circ}-100^{\circ} \mathrm{W} ; 36^{\circ}-52^{\circ} \mathrm{N}\right)$} & & & DJF & $-10.76 \mathrm{~W} \mathrm{~m}^{-2}$ & ns & $-9.83 \mathrm{~W} \mathrm{~m}^{-2}$ \\
\hline & & & & $-6.33 \%$ & ns & $-5.79 \%$ \\
\hline & & Direct & JJA & $-28.26 \mathrm{~W} \mathrm{~m}^{-2}$ & $-5.10 \mathrm{~W} \mathrm{~m}^{-2}$ & $-11.29 \mathrm{~W} \mathrm{~m}^{-2}$ \\
\hline & & & & $-26.22 \%$ & $-4.73 \%$ & $-10.48 \%$ \\
\hline & & & DJF & $-14.18 \mathrm{~W} \mathrm{~m}^{-2}$ & $-1.81 \mathrm{~W} \mathrm{~m}^{-2}$ & $-12.78 \mathrm{~W} \mathrm{~m}^{-2}$ \\
\hline & & & & $-35.00 \%$ & $-4.47 \%$ & $-31.55 \%$ \\
\hline & & Diffuse & JJA & $13.62 \mathrm{~W} \mathrm{~m}^{-2}$ & $1.85 \mathrm{~W} \mathrm{~m}^{-2}$ & $6.53 \mathrm{~W} \mathrm{~m}^{-2}$ \\
\hline & & & & $8.91 \%$ & $1.27 \%$ & $4.27 \%$ \\
\hline & & & DJF & $3.42 \mathrm{~W} \mathrm{~m}^{-2}$ & ns & $2.95 \mathrm{~W} \mathrm{~m}^{-2}$ \\
\hline & & & & $2.65 \%$ & ns & $2.28 \%$ \\
\hline & Canopy Temperature & & JJA & $-0.66 \mathrm{~K}$ & $-0.47 \mathrm{~K}$ & $-0.40 \mathrm{~K}$ \\
\hline & & & & $-0.22 \%$ & $-0.16 \%$ & $-0.14 \%$ \\
\hline & & & DJF & ns & ns & ns \\
\hline & & & & $\mathrm{ns}$ & ns & ns \\
\hline & GPP & & JJA & $0.54 \mathrm{Pg} \mathrm{Cyr}^{-1}$ & ns & $0.21 \mathrm{Pg} \mathrm{Cyr}^{-1}$ \\
\hline & & & & $5.83 \%$ & $\mathrm{~ns}$ & $2.32 \%$ \\
\hline & & & DJF & ns & $\mathrm{ns}$ & ns \\
\hline & & & & ns & ns & ns \\
\hline & Isoprene & & JJA & ns & ns & ns \\
\hline & & & & ns & ns & ns \\
\hline & & & DJF & ns & ns & ns \\
\hline & & & & $\mathrm{ns}$ & ns & ns \\
\hline
\end{tabular}


Table S3. Continued.

\begin{tabular}{|c|c|c|c|c|c|c|}
\hline Region & Variable & & Season & SimCTRL - SimNOant & SimCTRL - SimNObb & SimCTRL - SimNOind \\
\hline Eurasia & SW VIS Solar Radiation & Total & JJA & $-14.29 \mathrm{~W} \mathrm{~m}^{-2}$ & $-2.09 \mathrm{~W} \mathrm{~m}^{-2}$ & $-11.42 \mathrm{~W} \mathrm{~m}^{-2}$ \\
\hline \multirow[t]{23}{*}{$\left(-10^{\circ} \mathrm{W}-80^{\circ} \mathrm{E} ; 40^{\circ}-65^{\circ} \mathrm{N}\right)$} & & & & $-5.30 \%$ & $-0.78 \%$ & $-4.24 \%$ \\
\hline & & & DJF & $-7.79 \mathrm{~W} \mathrm{~m}^{-2}$ & ns & $-7.18 \mathrm{~W} \mathrm{~m}^{-2}$ \\
\hline & & & & $-6.26 \%$ & $\mathrm{~ns}$ & $-5.77 \%$ \\
\hline & & Direct & JJA & $-26.29 \mathrm{~W} \mathrm{~m}^{-2}$ & $-4.22 \mathrm{~W} \mathrm{~m}^{-2}$ & $-20.19 \mathrm{~W} \mathrm{~m}^{-2}$ \\
\hline & & & & $-26.77 \%$ & $-4.30 \%$ & $-20.57 \%$ \\
\hline & & & DJF & $-7.98 \mathrm{~W} \mathrm{~m}^{-2}$ & ns & $-7.44 \mathrm{~W} \mathrm{~m}^{-2}$ \\
\hline & & & & $-39.76 \%$ & ns & $-37.10 \%$ \\
\hline & & Diffuse & JJA & $12.00 \mathrm{~W} \mathrm{~m}^{-2}$ & $2.14 \mathrm{~W} \mathrm{~m}^{-2}$ & $8.77 \mathrm{~W} \mathrm{~m}^{-2}$ \\
\hline & & & & $7.01 \%$ & $1.25 \%$ & $5.13 \%$ \\
\hline & & & DJF & ns & ns & ns \\
\hline & & & & ns & ns & ns \\
\hline & Canopy Temperature & & JJA & $-0.46 \mathrm{~K}$ & ns & $-0.32 \mathrm{~K}$ \\
\hline & & & & $-0.16 \%$ & ns & $-0.11 \%$ \\
\hline & & & DJF & ns & ns & ns \\
\hline & & & & ns & $\mathrm{ns}$ & ns \\
\hline & GPP & & JJA & $1.84 \mathrm{Pg} \mathrm{Cyr}^{-1}$ & $0.48 \mathrm{Pg} \mathrm{Cyr}^{-1}$ & $1.06 \mathrm{Pg} \mathrm{Cyr}^{-1}$ \\
\hline & & & & $5.89 \%$ & $1.54 \%$ & $3.41 \%$ \\
\hline & & & DJF & ns & ns & ns \\
\hline & & & & ns & ns & ns \\
\hline & Isoprene & & JJA & $-1.63 \mathrm{Tg} \mathrm{Cyr}^{-1}$ & $\mathrm{~ns}$ & $-2.43 \mathrm{Tg} \mathrm{Cyr}^{-1}$ \\
\hline & & & & $-2.21 \%$ & $\mathrm{~ns}$ & $-3.29 \%$ \\
\hline & & & DJF & ns & ns & ns \\
\hline & & & & ns & ns & ns \\
\hline \multirow{24}{*}{$\begin{array}{l}\text { North-eastern China } \\
\left(100^{\circ}-120^{\circ} \mathrm{E} ; 10^{\circ}-35^{\circ} \mathrm{N}\right)\end{array}$} & SW VIS Solar Radiation & Total & JJA & $-14.36 \mathrm{~W} \mathrm{~m}^{-2}$ & $\mathrm{~ns}$ & $-12.17 \mathrm{~W} \mathrm{~m}^{-2}$ \\
\hline & & & & $-6.41 \%$ & ns & $-5.43 \%$ \\
\hline & & & DJF & $-12.77 \mathrm{~W} \mathrm{~m}^{-2}$ & ns & $-11.01 \mathrm{~W} \mathrm{~m}^{-2}$ \\
\hline & & & & $-6.34 \%$ & $\mathrm{~ns}$ & $-5.47 \%$ \\
\hline & & Direct & JJA & $-22.36 \mathrm{~W} \mathrm{~m}^{-2}$ & ns & $-19.34 \mathrm{~W} \mathrm{~m}^{-2}$ \\
\hline & & & & $-27.43 \%$ & ns & $-23.79 \%$ \\
\hline & & & DJF & $-21.08 \mathrm{~W} \mathrm{~m}^{-2}$ & $\mathrm{~ns}$ & $-18.91 \mathrm{~W} \mathrm{~m}^{-2}$ \\
\hline & & & & $-30.76 \%$ & ns & $-27.59 \%$ \\
\hline & & Diffuse & JJA & $8.00 \mathrm{~W} \mathrm{~m}^{-2}$ & ns & $7.22 \mathrm{~W} \mathrm{~m}^{-2}$ \\
\hline & & & & $5.61 \%$ & $\mathrm{~ns}$ & $5.07 \%$ \\
\hline & & & DJF & $8.31 \mathrm{~W} \mathrm{~m}^{-2}$ & $1.78 \mathrm{~W} \mathrm{~m}^{-2}$ & $7.90 \mathrm{~W} \mathrm{~m}^{-2}$ \\
\hline & & & & $6.26 \%$ & $1.34 \%$ & $5.95 \%$ \\
\hline & Canopy Temperature & & JJA & $-0.35 \mathrm{~K}$ & ns & ns \\
\hline & & & & $-0.12 \%$ & ns & ns \\
\hline & & & DJF & ns & $\mathrm{ns}$ & ns \\
\hline & & & & ns & ns & ns \\
\hline & GPP & & JJA & $0.26 \mathrm{Pg} \mathrm{Cyr}^{-1}$ & ns & $0.20 \mathrm{Pg} \mathrm{Cyr}^{-1}$ \\
\hline & & & & $3.03 \%$ & ns & $2.37 \%$ \\
\hline & & & DJF & ns & ns & ns \\
\hline & & & & ns & ns & ns \\
\hline & Isoprene & & JJA & $-1.12 \mathrm{Tg} \mathrm{Cyr}^{-1}$ & $\mathrm{~ns}$ & $-1.04 \mathrm{Tg} \mathrm{Cyr}^{-1}$ \\
\hline & & & & $-3.85 \%$ & ns & $-3.57 \%$ \\
\hline & & & DJF & $-0.60 \mathrm{Tg} \mathrm{C} \mathrm{yr}^{-1}$ & ns & $-0.48 \mathrm{Tg} \mathrm{Cyr}^{-1}$ \\
\hline & & & & $-7.15 \%$ & ns & $-5.77 \%$ \\
\hline
\end{tabular}


Table S4. As Table S3 for absolute and percent changes in seasonal average shortwave visible (SW VIS) solar radiation, canopy temperature, gross primary productivity (GPP), and isoprene emission in: north-western Amazon Basin and central Africa (green boxes on Fig. 1a in the manuscript). Boreal summer (JJA), autumn (SON) and winter (DJF) seasonal averages are reported.

\begin{tabular}{|c|c|c|c|c|c|c|}
\hline \multirow{2}{*}{$\begin{array}{l}\text { Region } \\
\text { North-western }\end{array}$} & \multicolumn{2}{|l|}{ Variable } & \multirow{2}{*}{$\begin{array}{l}\text { Season } \\
\text { JJA }\end{array}$} & \multirow{2}{*}{$\begin{array}{c}\text { SimCTRL }- \text { SimNOant } \\
-5.21 \mathrm{~W} \mathrm{~m}^{-2}\end{array}$} & \multirow{2}{*}{$\begin{array}{c}\text { SimCTRL }- \text { SimNObb } \\
-3.33 \mathrm{~W} \mathrm{~m}^{-2}\end{array}$} & \multirow{2}{*}{$\begin{array}{c}\text { SimCTRL }- \text { SimNOind } \\
-3.59 \mathrm{~W} \mathrm{~m}^{-2}\end{array}$} \\
\hline & SW VIS Solar Radiation & Total & & & & \\
\hline Amazon Basin & & & & $-2.36 \%$ & $-1.51 \%$ & $-1.63 \%$ \\
\hline \multirow[t]{34}{*}{$\left(73^{\circ}-65^{\circ} \mathrm{W} ; 5^{\circ} \mathrm{S}-5^{\circ} \mathrm{N}\right)$} & & & SON & $-5.18 \mathrm{~W} \mathrm{~m}^{-2}$ & $-2.46 \mathrm{~W} \mathrm{~m}^{-2}$ & $-2.18 \mathrm{~W} \mathrm{~m}^{-2}$ \\
\hline & & & & $-2.32 \%$ & $-1.10 \%$ & $-0.98 \%$ \\
\hline & & & DJF & $-3.47 \mathrm{~W} \mathrm{~m}^{-2}$ & $-2.11 \mathrm{~W} \mathrm{~m}^{-2}$ & $-1.96 \mathrm{~W} \mathrm{~m}^{-2}$ \\
\hline & & & & $-1.53 \%$ & $-0.93 \%$ & $-0.87 \%$ \\
\hline & & Direct & JJA & $-9.98 \mathrm{~W} \mathrm{~m}^{-2}$ & $-5.87 \mathrm{~W} \mathrm{~m}^{-2}$ & $-6.87 \mathrm{~W} \mathrm{~m}^{-2}$ \\
\hline & & & & $-10.12 \%$ & $-5.95 \%$ & $-6.96 \%$ \\
\hline & & & SON & $-9.75 \mathrm{~W} \mathrm{~m}^{-2}$ & $-4.66 \mathrm{~W} \mathrm{~m}^{-2}$ & $-4.80 \mathrm{~W} \mathrm{~m}^{-2}$ \\
\hline & & & & $-9.92 \%$ & $-4.74 \%$ & $-4.88 \%$ \\
\hline & & & DJF & $-5.93 \mathrm{~W} \mathrm{~m}^{-2}$ & $-3.45 \mathrm{~W} \mathrm{~m}^{-2}$ & $-3.94 \mathrm{~W} \mathrm{~m}^{-2}$ \\
\hline & & & & $-6.05 \%$ & $-3.52 \%$ & $-4.01 \%$ \\
\hline & & Diffuse & JJA & $4.76 \mathrm{~W} \mathrm{~m}^{-2}$ & $2.54 \mathrm{~W} \mathrm{~m}^{-2}$ & $3.28 \mathrm{~W} \mathrm{~m}^{-2}$ \\
\hline & & & & $3.89 \%$ & $2.08 \%$ & $2.68 \%$ \\
\hline & & & SON & $4.57 \mathrm{~W} \mathrm{~m}^{-2}$ & $2.20 \mathrm{~W} \mathrm{~m}^{-2}$ & $2.62 \mathrm{~W} \mathrm{~m}^{-2}$ \\
\hline & & & & $3.66 \%$ & $1.76 \%$ & $2.10 \%$ \\
\hline & & & DJF & $2.47 \mathrm{~W} \mathrm{~m}^{-2}$ & $1.34 \mathrm{~W} \mathrm{~m}^{-2}$ & $1.97 \mathrm{~W} \mathrm{~m}^{-2}$ \\
\hline & & & & $1.93 \%$ & $1.05 \%$ & $1.54 \%$ \\
\hline & Canopy Temperature & & JJA & $\mathrm{ns}$ & $-0.53 \mathrm{~K}$ & ns \\
\hline & & & & ns & $-0.18 \%$ & ns \\
\hline & & & SON & $-0.34 \mathrm{~K}$ & $-0.58 \mathrm{~K}$ & ns \\
\hline & & & & $-0.19 \%$ & $-0.19 \%$ & $\mathrm{~ns}$ \\
\hline & & & DJF & $-0.09 \mathrm{~K}$ & $-0.09 \mathrm{~K}$ & $-0.07 \mathrm{~K}$ \\
\hline & & & & $-0.03 \%$ & $-0.03 \%$ & $-0.02 \%$ \\
\hline & GPP & & JJA & $0.09 \mathrm{Pg} \mathrm{Cyr}^{-1}$ & $0.16 \mathrm{Pg} \mathrm{Cyr}^{-1}$ & $0.07 \mathrm{Pg} \mathrm{Cyr}^{-1}$ \\
\hline & & & & $2.97 \%$ & $5.25 \%$ & $2.39 \%$ \\
\hline & & & SON & $0.11 \mathrm{PgC} \mathrm{yr}^{-1}$ & $0.17 \mathrm{Pg} \mathrm{C} \mathrm{yr}^{-1}$ & $0.08 \mathrm{Pg} \mathrm{C} \mathrm{yr}^{-1}$ \\
\hline & & & & $4.18 \%$ & $6.43 \%$ & $2.98 \%$ \\
\hline & & & DJF & $0.04 \mathrm{Pg} \mathrm{C} \mathrm{yr}^{-1}$ & $0.03 \mathrm{Pg} \mathrm{C} \mathrm{yr}^{-1}$ & $0.03 \mathrm{Pg} \mathrm{C} \mathrm{yr}^{-1}$ \\
\hline & & & & $1.36 \%$ & $1.15 \%$ & $1.14 \%$ \\
\hline & Isoprene & & JJA & ns & $0.55 \mathrm{Tg} \mathrm{C} \mathrm{yr}^{-1}$ & ns \\
\hline & & & & ns & $3.46 \%$ & ns \\
\hline & & & SON & $\mathrm{ns}$ & $1.00 \mathrm{Tg} \mathrm{Cyr}^{-1}$ & $\mathrm{~ns}$ \\
\hline & & & & ns & $6.66 \%$ & $\mathrm{~ns}$ \\
\hline & & & DJF & ns & ns & ns \\
\hline & & & & $\mathrm{ns}$ & $\mathrm{ns}$ & $\mathrm{ns}$ \\
\hline
\end{tabular}


Table S4. Continued.

\begin{tabular}{|c|c|c|c|c|c|c|}
\hline \multirow{3}{*}{$\begin{array}{l}\text { Region } \\
\text { Central Africa } \\
\left(10^{\circ}-25^{\circ} \mathrm{E} ; 10^{\circ} \mathrm{S}-5^{\circ} \mathrm{N}\right)\end{array}$} & \multicolumn{2}{|l|}{ Variable } & \multirow{3}{*}{$\begin{array}{l}\text { Season } \\
\text { JJA }\end{array}$} & \multirow{2}{*}{$\begin{array}{c}\text { SimCTRL }- \text { SimNOant } \\
-28.22 \mathrm{~W} \mathrm{~m}^{-2}\end{array}$} & \multirow{2}{*}{$\begin{array}{c}\text { SimCTRL }- \text { SimNObb } \\
-24.60 \mathrm{~W} \mathrm{~m}^{-2}\end{array}$} & \multirow{2}{*}{$\begin{array}{c}\text { SimCTRL }- \text { SimNOind } \\
-13.78 \mathrm{~W} \mathrm{~m}^{-2}\end{array}$} \\
\hline & SW VIS Solar Radiation & Total & & & & \\
\hline & & & & $-14.34 \%$ & $-12.50 \%$ & $-7.00 \%$ \\
\hline & & & SON & $-10.71 \mathrm{~W} \mathrm{~m}^{-2}$ & $-7.58 \mathrm{~W} \mathrm{~m}^{-2}$ & $-4.34 \mathrm{~W} \mathrm{~m}^{-2}$ \\
\hline & & & & $-5.23 \%$ & $-3.70 \%$ & $-2.12 \%$ \\
\hline & & & DJF & $-22.96 \mathrm{~W} \mathrm{~m}^{-2}$ & $-20.52 \mathrm{~W} \mathrm{~m}^{-2}$ & $-11.71 \mathrm{~W} \mathrm{~m}^{-2}$ \\
\hline & & & & $-11.75 \%$ & $-10.50 \%$ & $-5.99 \%$ \\
\hline & & Direct & JJA & $-44.16 \mathrm{~W} \mathrm{~m}^{-2}$ & $-36.52 \mathrm{~W} \mathrm{~m}^{-2}$ & $-18.88 \mathrm{~W} \mathrm{~m}^{-2}$ \\
\hline & & & & $-68.66 \%$ & $-56.75 \%$ & $-29.35 \%$ \\
\hline & & & SON & $-17.57 \mathrm{~W} \mathrm{~m}^{-2}$ & $-11.03 \mathrm{~W} \mathrm{~m}^{-2}$ & $-7.03 \mathrm{~W} \mathrm{~m}^{-2}$ \\
\hline & & & & $-22.68 \%$ & $-14.25 \%$ & $-9.08 \%$ \\
\hline & & & DJF & $-24.23 \mathrm{~W} \mathrm{~m}^{-2}$ & $-19.43 \mathrm{~W} \mathrm{~m}^{-2}$ & $-10.35 \mathrm{~W} \mathrm{~m}^{-2}$ \\
\hline & & & & $-38.18 \%$ & $-30.60 \%$ & $-16.31, \%$ \\
\hline & & Diffuse & JJA & $15.93 \mathrm{~W} \mathrm{~m}^{-2}$ & $11.91 \mathrm{~W} \mathrm{~m}^{-2}$ & $5.10 \mathrm{~W} \mathrm{~m}^{-2}$ \\
\hline & & & & $12.02 \%$ & $8.99 \%$ & $3.85 \%$ \\
\hline & & & SON & $6.86 \mathrm{~W} \mathrm{~m}^{-2}$ & $3.45 \mathrm{~W} \mathrm{~m}^{-2}$ & $2.70 \mathrm{~W} \mathrm{~m}^{-2}$ \\
\hline & & & & $5.39 \%$ & $2.17 \%$ & $2.12 \%$ \\
\hline & & & DJF & $1.27 \mathrm{~W} \mathrm{~m}^{-2}$ & $-1.09 \mathrm{~W} \mathrm{~m}^{-2}$ & $-1.36 \mathrm{~W} \mathrm{~m}^{-2}$ \\
\hline & & & & $0.96 \%$ & $-0.83 \%$ & $-1.03 \%$ \\
\hline & Canopy Temperature & & JJA & $-0.36 \mathrm{~K}$ & $-0.32 \mathrm{~K}$ & $-0.16 \mathrm{~K}$ \\
\hline & & & & $-0.12 \%$ & $-0.11 \%$ & $-0.05 \%$ \\
\hline & & & SON & $-0.11 \mathrm{~K}$ & ns & ns \\
\hline & & & & -0.06 & ns & ns \\
\hline & & & DJF & $-0.34 \mathrm{~K}$ & $-0.33 \mathrm{~K}$ & $-0.13 \mathrm{~K}$ \\
\hline & & & & $-0.11 \%$ & $-0.11 \%$ & $-0.04 \%$ \\
\hline & GPP & & JJA & $0.12 \mathrm{Pg} \mathrm{Cyr}^{-1}$ & $0.10 \mathrm{Pg} \mathrm{Cyr}^{-1}$ & ns \\
\hline & & & & $2.66 \%$ & $2.22 \%$ & ns \\
\hline & & & SON & $0.14 \mathrm{Pg} \mathrm{Cyr}^{-1}$ & $0.08 \mathrm{Pg} \mathrm{Cyr}^{-1}$ & ns \\
\hline & & & & $2.3 \%$ & $1.43 \%$ & ns \\
\hline & & & DJF & $0.14 \mathrm{Pg} \mathrm{C}_{\mathrm{yr}}{ }^{-1}$ & $0.17 \mathrm{Pg} \mathrm{Cyr}^{-1}$ & ns \\
\hline & & & & $1.97 \%$ & $2.33 \%$ & ns \\
\hline & Isoprene & & JJA & ns & ns & ns \\
\hline & & & & $\mathrm{ns}$ & $\mathrm{ns}$ & ns \\
\hline & & & SON & ns & ns & ns \\
\hline & & & & ns & $\mathrm{ns}$ & ns \\
\hline & & & DJF & $-1.99 \mathrm{Tg} \mathrm{C} \mathrm{yr}^{-1}$ & $-1.16 \mathrm{Tg} \mathrm{Cyr}^{-1}$ & $-1.06 \mathrm{Tg} \mathrm{C} \mathrm{yr}^{-1}$ \\
\hline & & & & $-6.03 \%$ & $-3.52 \%$ & $-3.21 \%$ \\
\hline
\end{tabular}



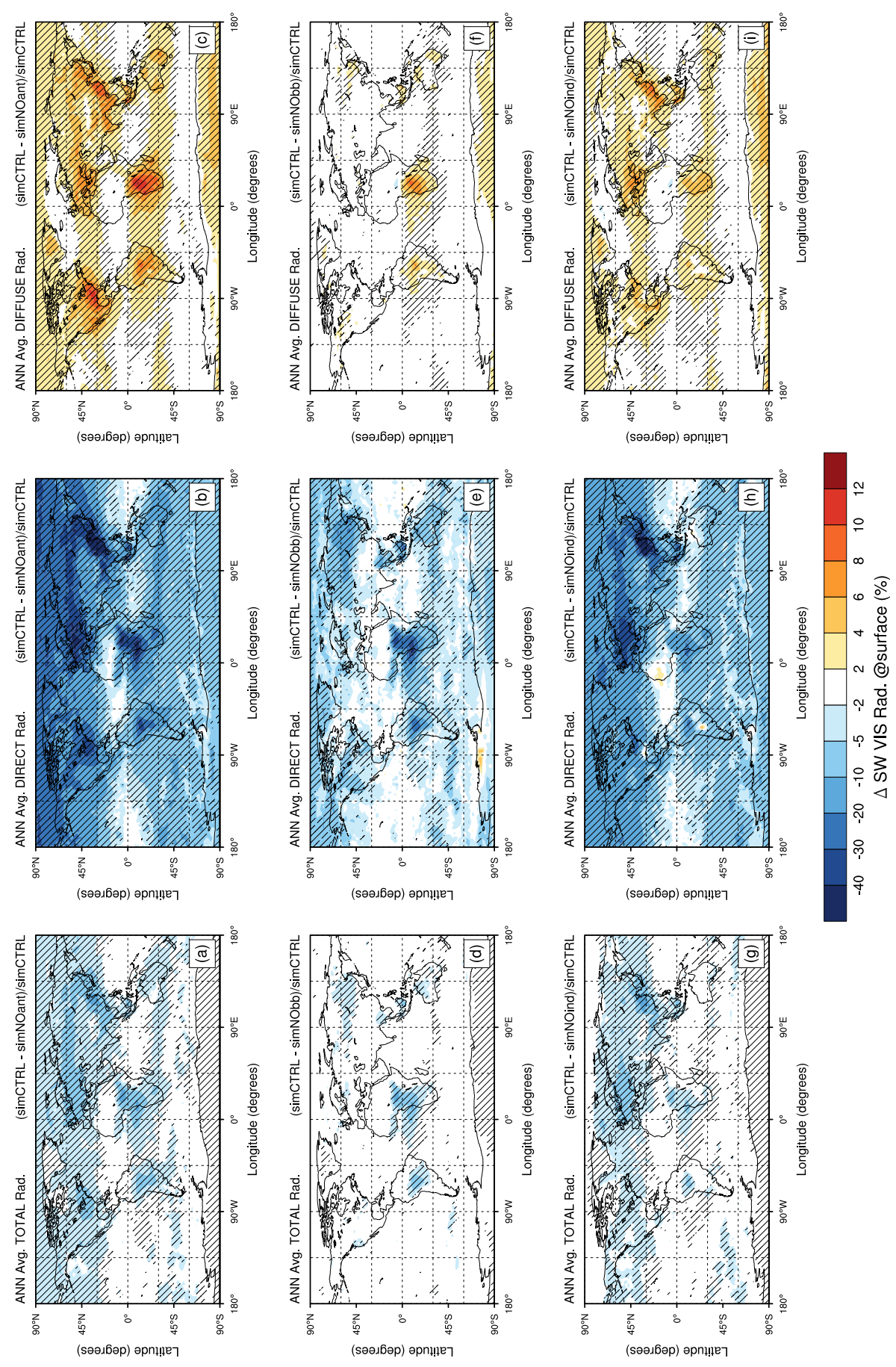

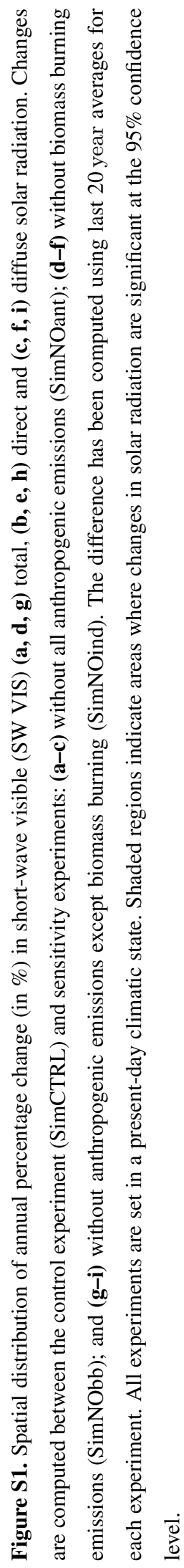



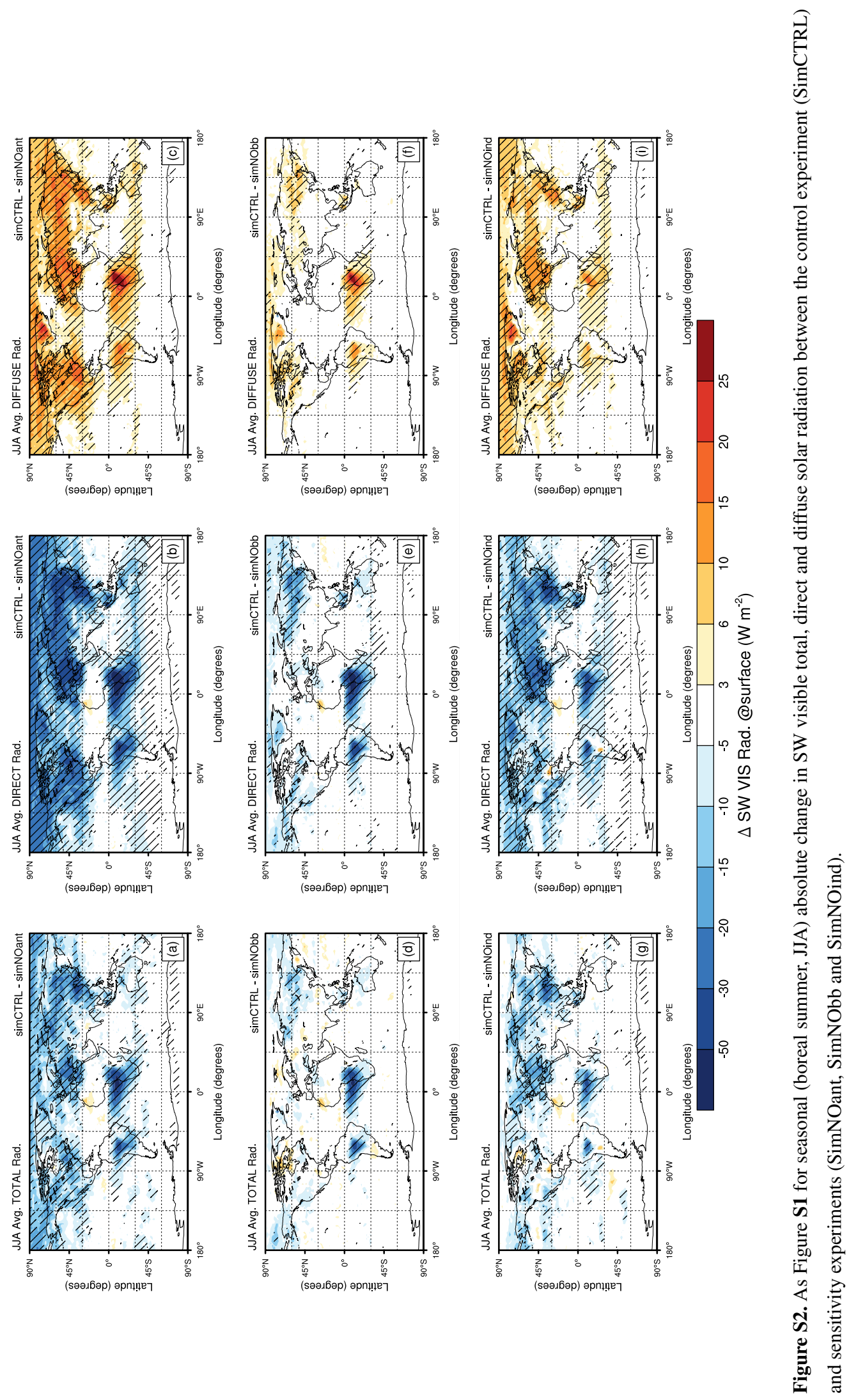

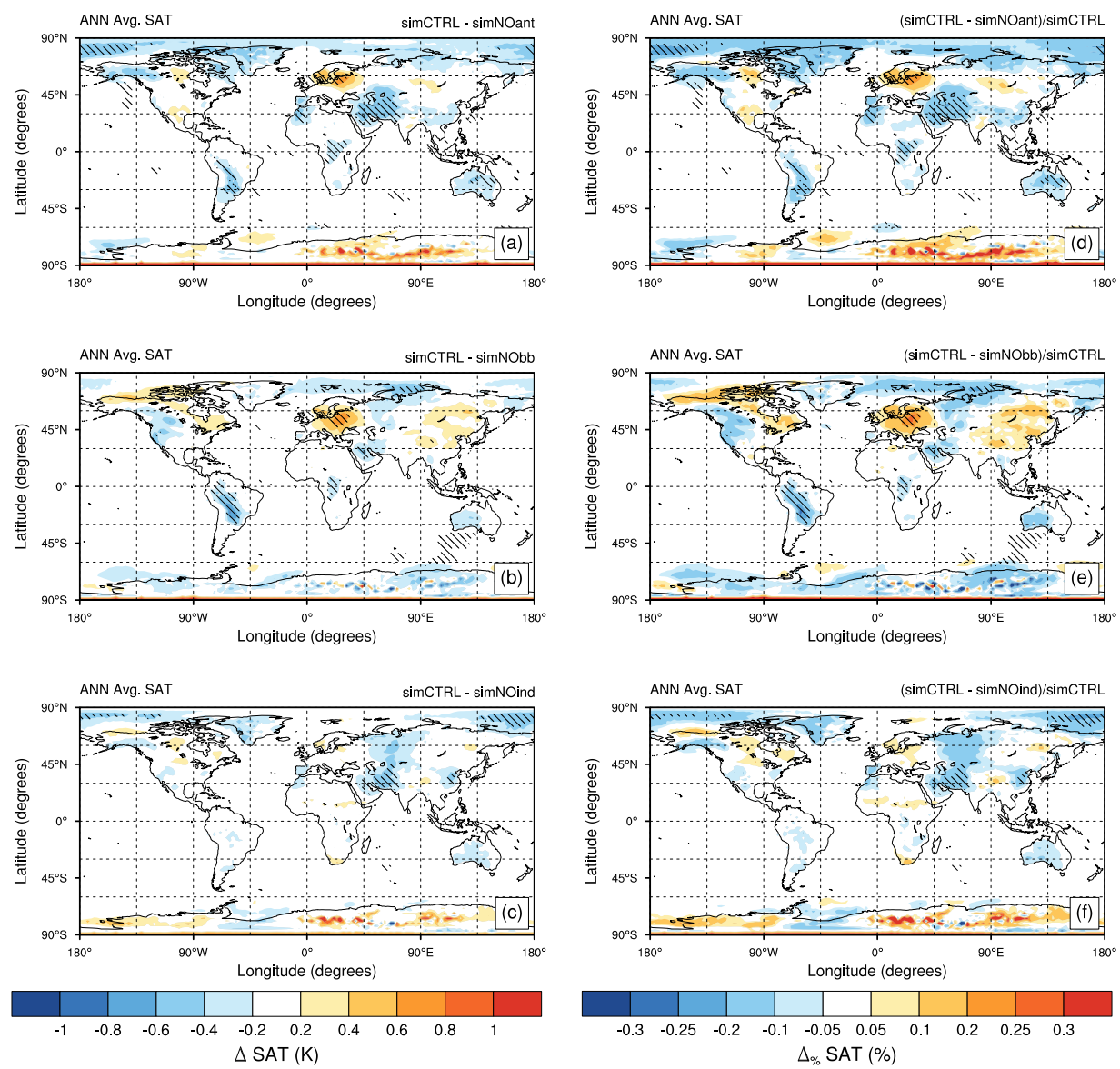

Figure S3. Spatial distribution of annual absolute (in K, left column panels) and percentage change (in \%, right column panels) in surface atmospheric temperature (SAT) between the control experiment (SimCTRL) and sensitivity experiments: (a) and (d) without all anthropogenic emissions (SimNOant); (b) and (e) without biomass burning emissions (SimNObb); (c) and (f) without anthropogenic emissions except biomass burning (SimNOind). The difference has been computed using last 20 year averages for each experiment. All experiments are set in a present-day climatic state. Shaded regions indicate areas where changes are significant at the 95\% confidence level. 

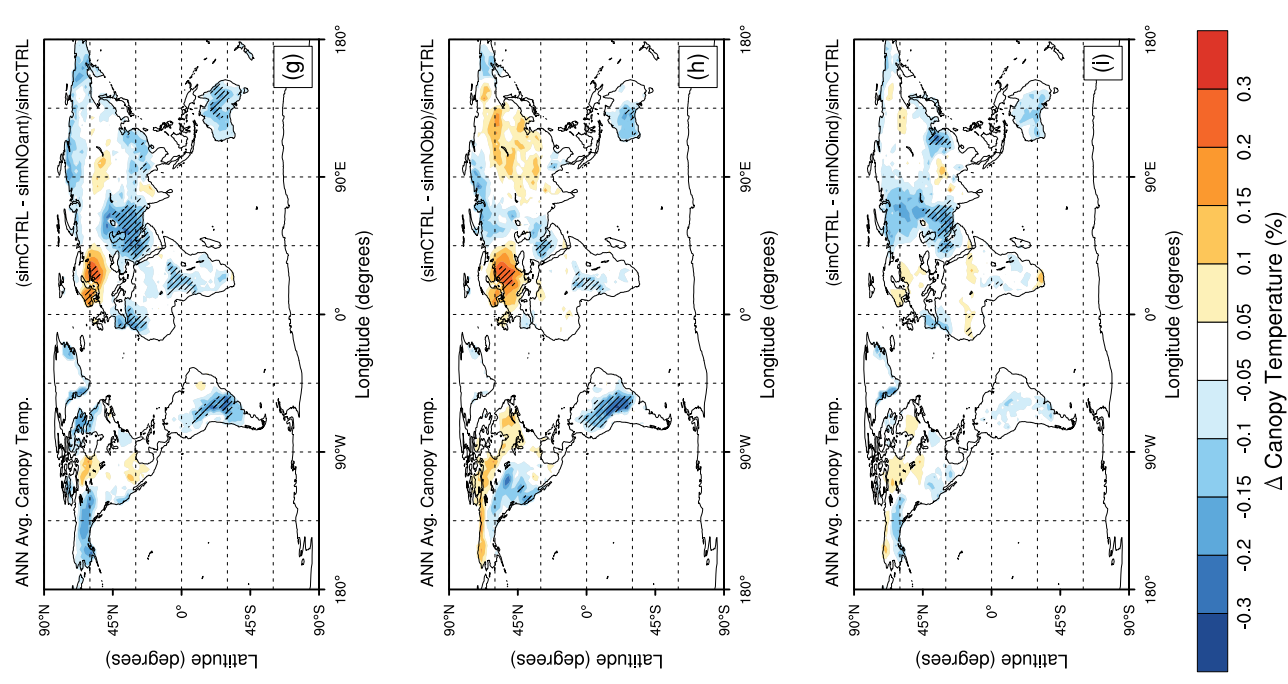

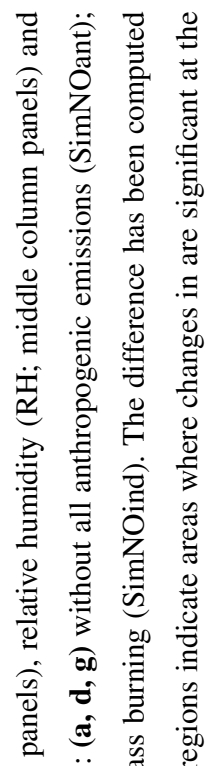
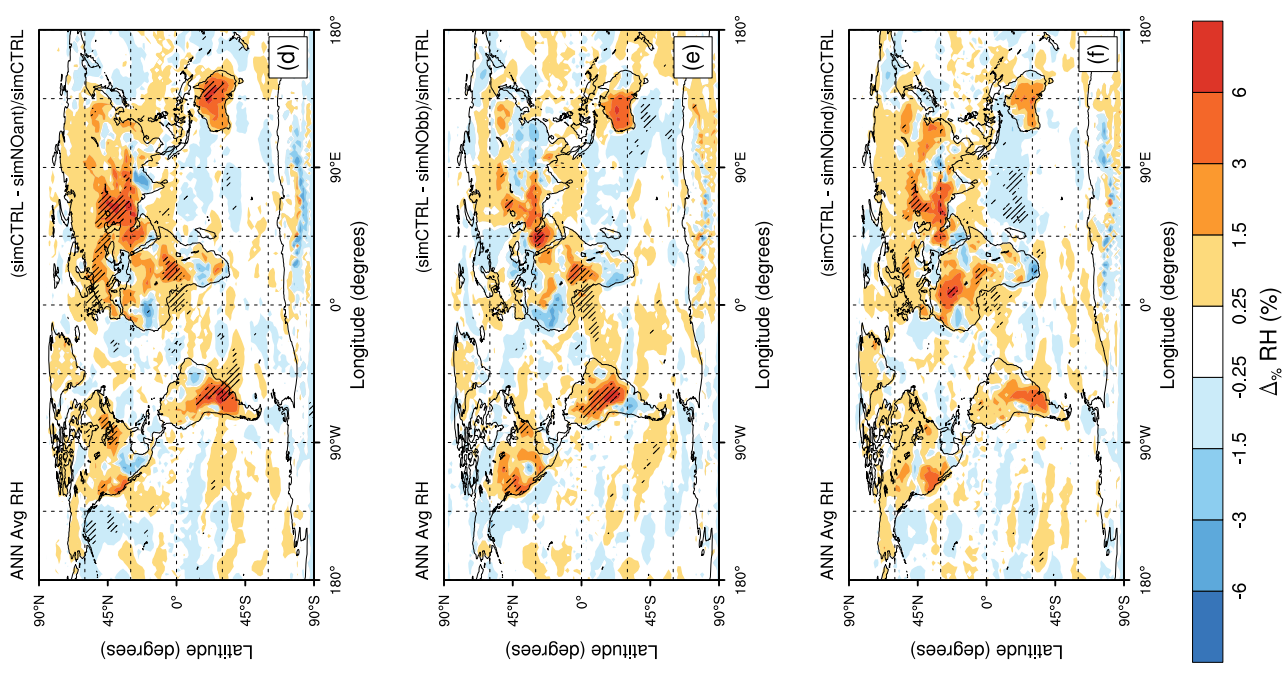

声总苛

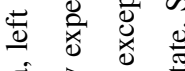

密 胥

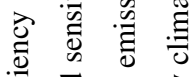

记

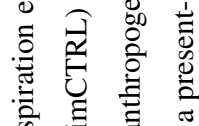

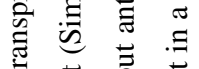

声苛总品

के 3

可 खें
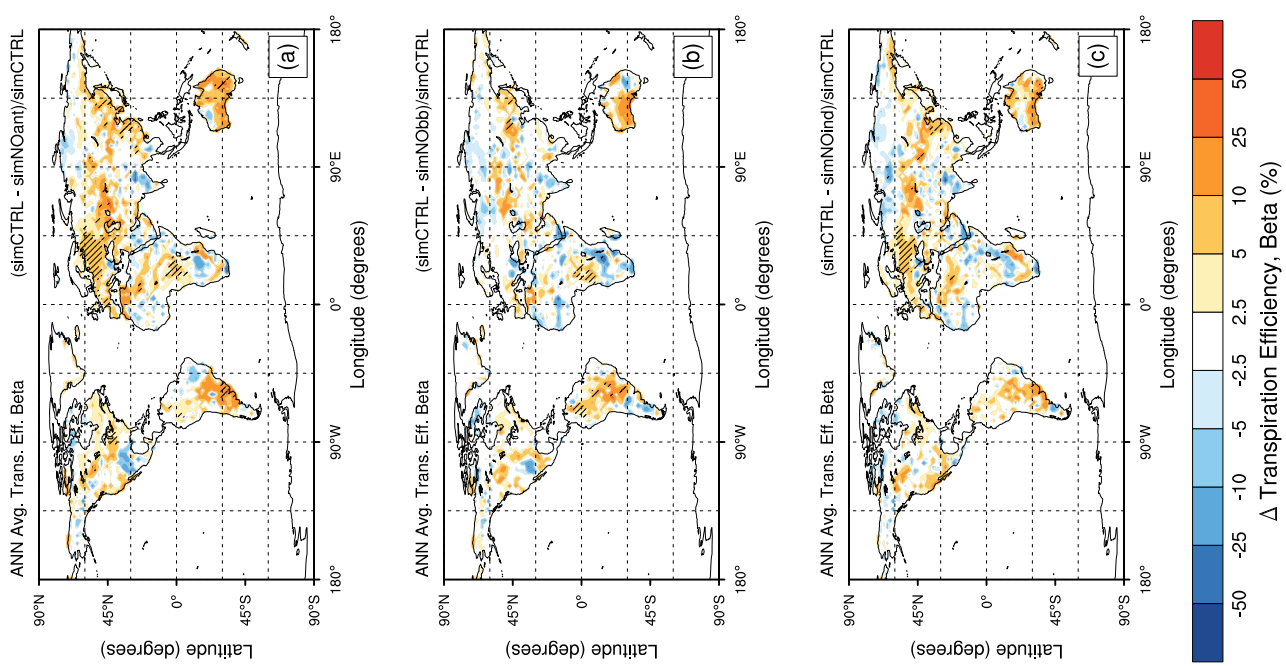

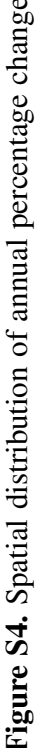
享 密 严文

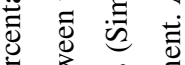
蓠

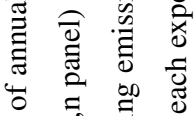
홍ㅎㅀ

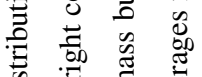
量 点

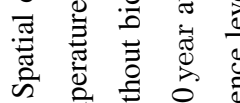

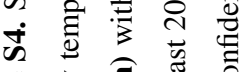

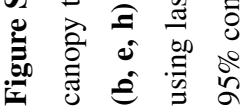



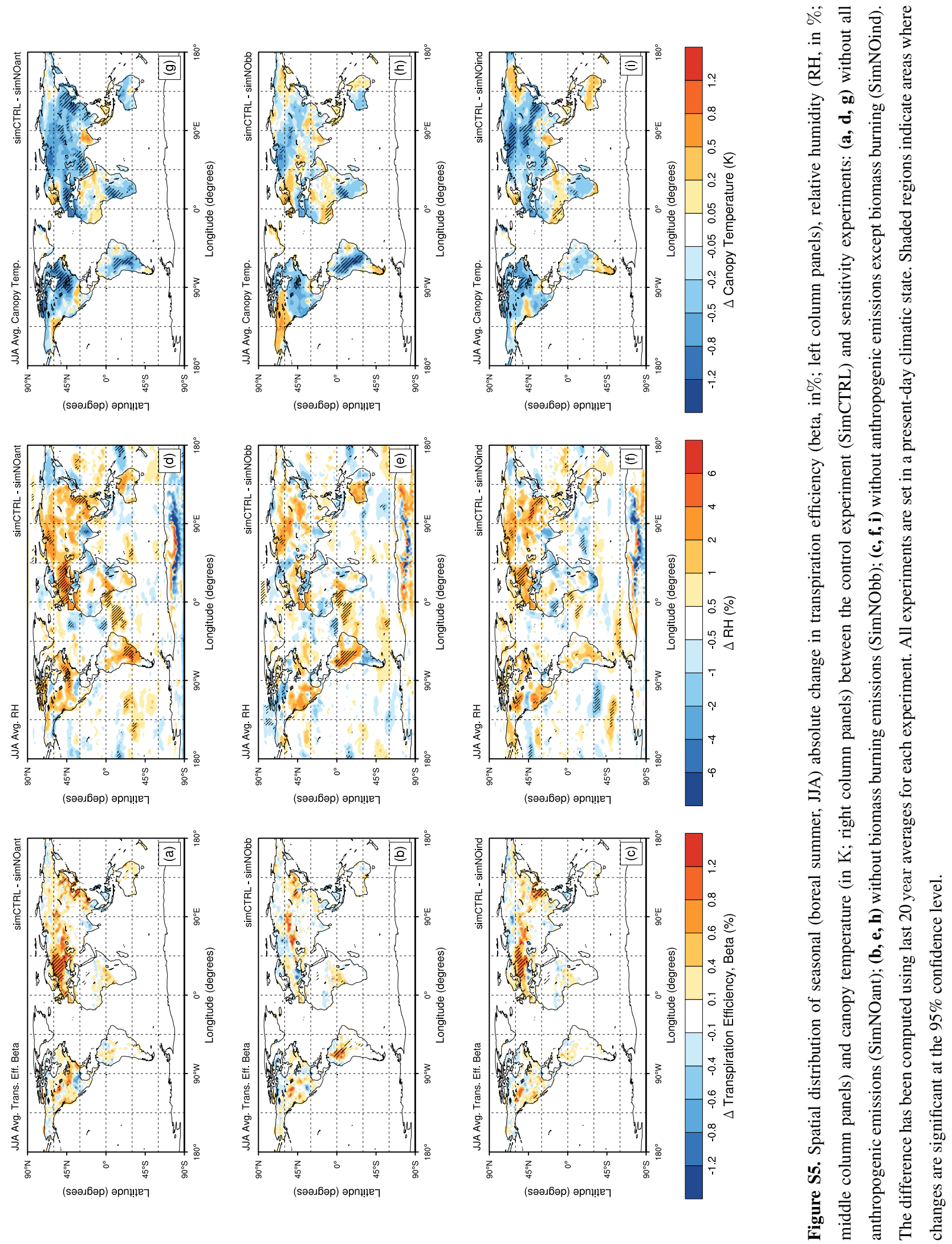

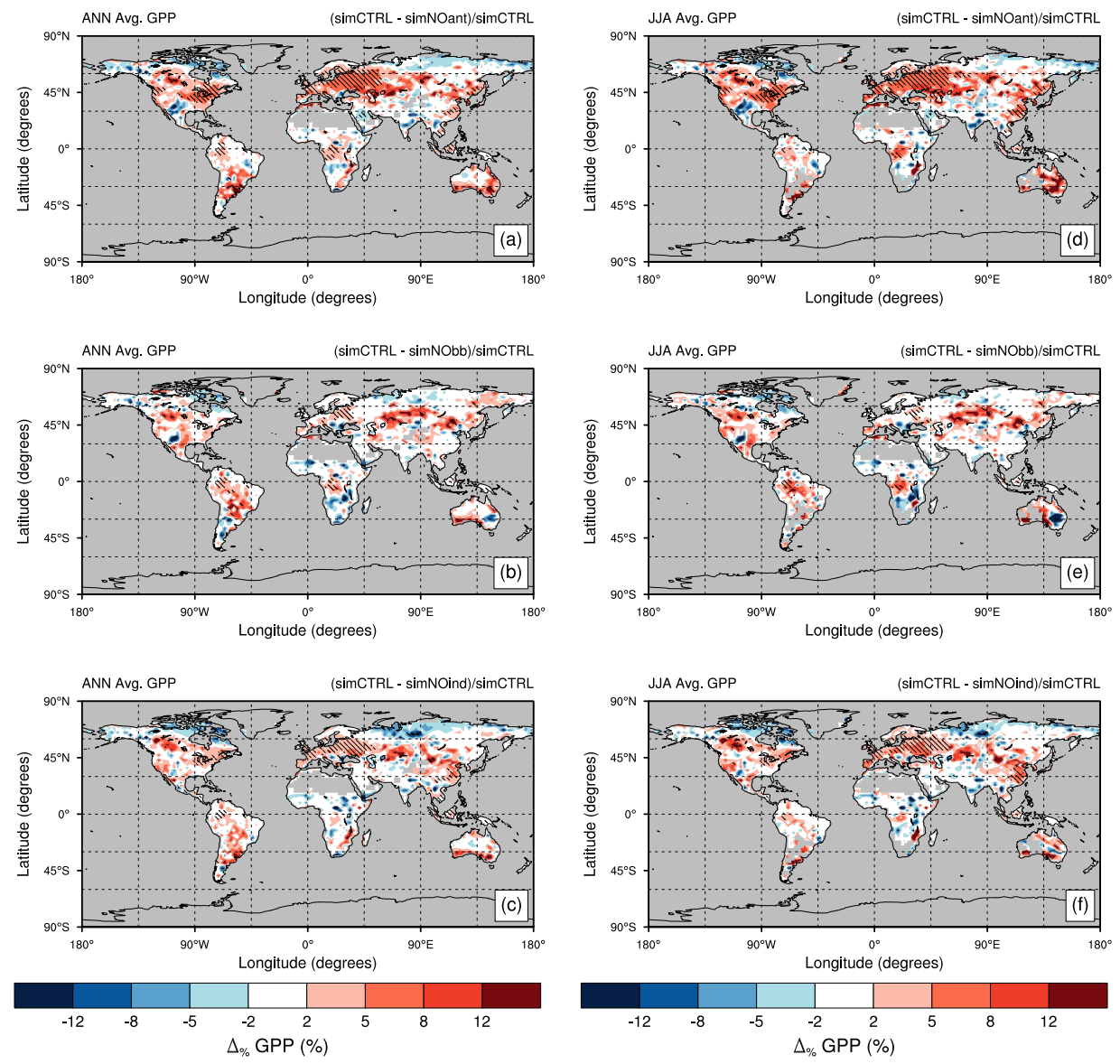

Figure S6. Spatial distribution of annual (left column panels) and seasonal (boreal summer, JJA; right column panels) percentage change (in \%) in Gross Primary Productivity (GPP) between the control experiment ("SimCTRL") and sensitivity experiments: (a) and (d) without all anthropogenic emissions ("SimNOant"); (b) and (e) without biomass burning emissions ("SimNObb"); (c) and (f) without anthropogenic emissions except biomass burning ("SimNOind"). The difference has been computed using last 20 year averages for each experiment. All experiments are set in a present-day climatic state. Shaded regions indicate areas where changes in GPP are significant at the $95 \%$ confidence level. 

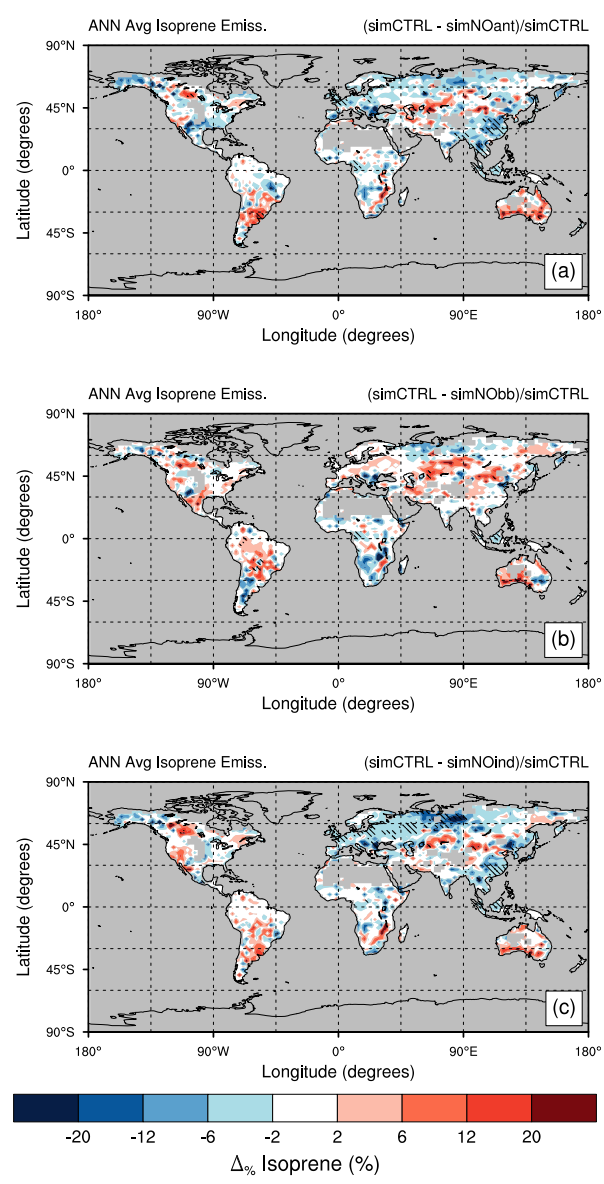

Figure S7. Spatial distribution of annual percentage change (in \%) in isoprene emission between the control experiment (SimCTRL) and sensitivity experiments: (a) without all anthropogenic emissions (SimNOant); (b) without biomass burning emissions (SimNObb); and (c) without anthropogenic emissions except biomass burning (SimNOind). The difference has been computed using last 20 year averages for each experiment. All experiments are set in a present-day climatic state. Shaded regions indicate areas where changes in isoprene emissions are significant at the $95 \%$ confidence level. 

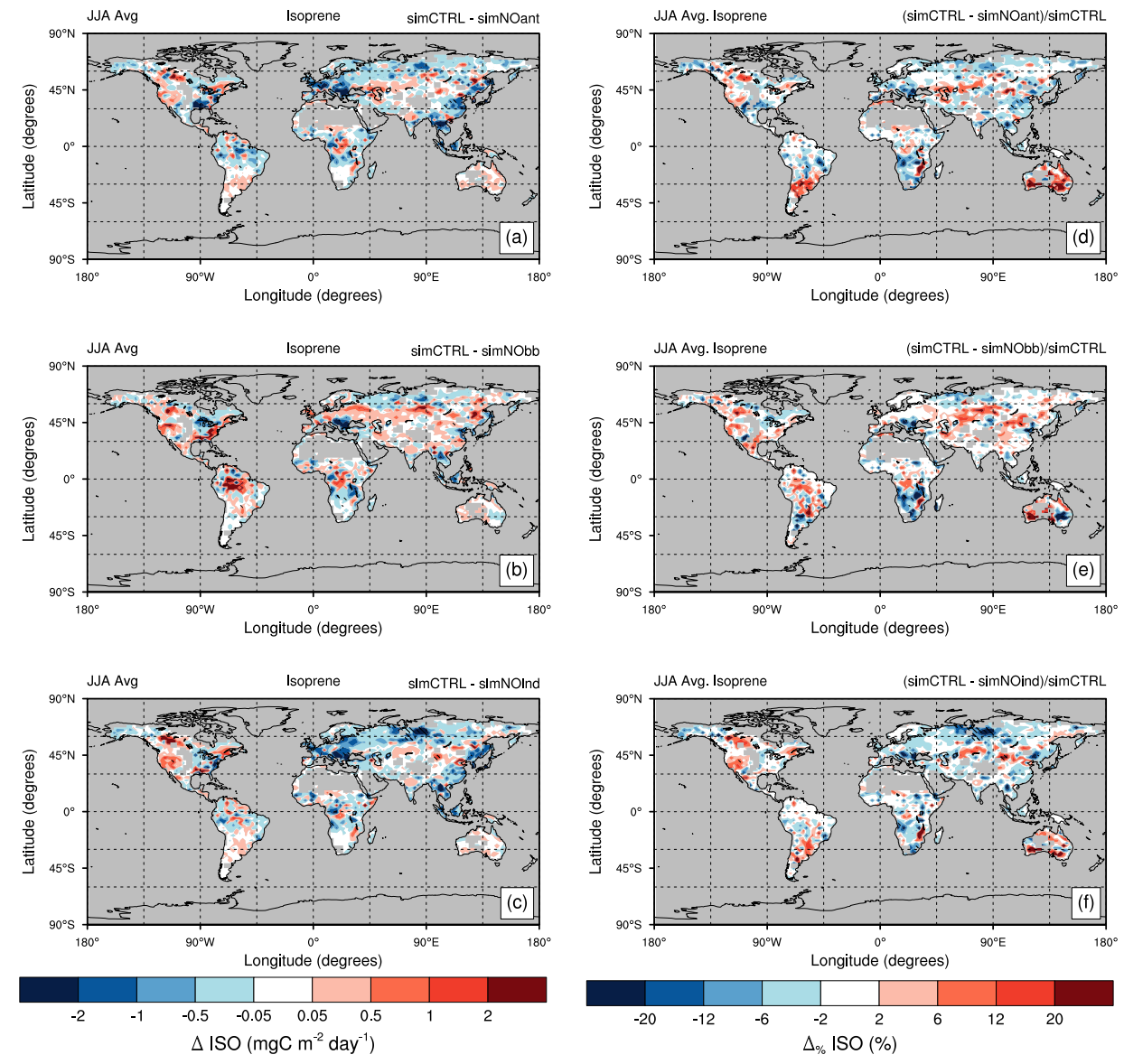

Figure S8. Spatial distribution of seasonal (boreal summer, JJA) absolute (in $\mathrm{mg} \mathrm{C} \mathrm{m}^{-2}$ day $^{-1}$ ) and percentage (in \%) change in isoprene emission between the control experiment (SimCTRL) and sensitivity experiments: (a) and (d) without all anthropogenic emissions (SimNOant); (b) and (e) without biomass burning emissions (SimNObb); (c) and (f) without anthropogenic emissions except biomass burning (SimNOind). The difference has been computed using last 20 year averages for each experiment. All experiments are set in a present-day climatic state. Shaded regions indicate areas where changes in isoprene emissions are significant at the $95 \%$ confidence level. 